\title{
Solar Surface Anisotropy effect on the Magnetic Field
}

\author{
Véronique Bommier
}

LESIA, Observatoire de Paris, CNRS-INSU-UMR8109, UPMC Univ. Paris 06, Université Paris Diderot-Paris 7; 5, Place Jules Janssen, 92190 Meudon, France

\begin{abstract}
Within the literature there are at least 15 references indicating that the horizontal magnetic flux does not exactly balance vertical flux in sunspots, leading to the surprising result that $\operatorname{div} \vec{B}$ would depart from zero. Intuitively, this has to be related to the stratification at the surface of the star, due to which horizontal and vertical typical lengths are different. This surface anisotropy results from gravity, but how does gravity influence the magnetic field? To answer this question, a scenario has been proposed in two recent publications, based on anisotropic Debye shielding. The presentation reported in this paper was devoted to investigate the possibility and causes of a non-zero div $\vec{B}$. A scaling law associated with the anisotropy is able to reestablish the nullity of $\operatorname{div} \vec{B}$, which would lead to a renewed MHD in the solar photosphere layer. An eventual observation in the laboratory is also reported.
\end{abstract}

Keywords. Sun: magnetic fields, Sun: photosphere, sunspots, MHD

\section{Introduction}

The determination of $\operatorname{div} \vec{B}$ from observations is a non-trivial task becauses this requires the availability of ambiguity-resolved magnetic field maps at least at two different depths in the solar atmosphere, and most often the ambiguity resolution is itself based on the $\operatorname{div} \vec{B}=0$ condition. This is the reason why, in the literature, one generally finds only partial gradient determination, either vertical or horizontal. Vertical gradient determinations are more frequent than the horizontal ones, the latter requiring the ambiguity resolution, and usually may be achieved in penumbrae only where the resolution may be accomplished via intuitive means alone for most sunspots . A detailed description of these observational results and associated methods may be found in Bommier (2013), and in a more compact manner in Bommier (2014). Remarkably, different observations obtained with different telescopes, spectral lines, and inversion methods agree without exception on a value of $3 \mathrm{G} / \mathrm{km}$ for the vertical gradient, and $0.3 \mathrm{G} / \mathrm{km}$ for the horizontal gradient (absolute values). This difference in value necessarily implies a non-zero div $\vec{B}$, but this was not so clear in reading the literature because the authors transformed horizontal gradients into vertical ones via $\operatorname{div} \vec{B}=0$. They then concluded that the values of the vertical gradient of the sunspot magnetic field are highly dispersed in the literature. Once the true value of the measured gradient is clarified by reading the article body, the remarkable result for uniform but different values of the horizontal and vertical gradients appears.

We obtained similar values from THEMIS observation of a $\delta$-spot on 13 September 2005. The ambiguity was solved by minimizing the scaled $\operatorname{div} \vec{B}$ as presented in the conclusion of this paper. Both horizontal and vertical gradients were obtained, with the values quoted above. The $\operatorname{div} \vec{B}$ departed so much from zero that it was possible to plot a map of $\operatorname{div} \vec{B}$ for this $\delta$-spot, which clearly displays the $\delta$-spot shape. This is 
Figure 3 of Bommier (2013). The observations were spectropolarimetric ones in two lines, Fe I 6301.5 and $6302.5 \AA$. We applied to them independent Milne-Edddington inversion with the UNNOFIT code (Bommier et al. 2007). These two lines belong to the same multiplet. As visible in Figure 4 of Khomenko \& Collados (2007), which is the result of a simulation, the difference in their height of formation remains very constant, even if the formation height varies due to the thermodynamical variations through the atmosphere. This is physically due to the fact that the two lines belong to the same multiplet, so that they are formed in a very similar manner, but their absorption coefficients differ by a theoretical factor of three, leading to different respective formation heights. We used the height difference directly measured by Faurobert et al. (2009), who applied a spatial phase shift technique to HINODE data taken out of disk center. They obtained $63.2 \pm 0.9$ $\mathrm{km}$ as the formation height difference, in the quiet Sun, in excellent agreement with 3D simulations of solar magneto-convection from Stein \& Nordlund and Uitenbroek's codes (Grec et al. 2010), which give $69 \mathrm{~km}$. As Figure 4 of Khomenko \& Collados (2007) shows that this difference would increase in active regions, with respect to the quiet Sun, we finally applied the value of $98 \mathrm{~km}$ in our data treatment. The ambiguity was removed by minimizing $|\operatorname{div} \vec{B}|+\left|J_{Z}\right|$, as recommended by Metcalf (1994). The necessity to add $\left|J_{Z}\right|$ to $|\operatorname{div} \vec{B}|$ comes from the fact that $|\operatorname{div} \vec{B}|$ has its own ambiguity and is then not totally sufficient. The technique we applied is close to the one of the ME0 code by Leka et al. (2009), except for the fact that the vertical extrapolation applied in ME0 to derive the vertical gradient was replaced by the observed vertical gradient from the two-line data. As the vertical and horizontal gradients finally differ, we applied to $|\operatorname{div} \vec{B}|$ the scaling described in the conclusion of this paper.

Puschmann et al. (2010) also obtained different vertical and horizontal gradients by analyzing a portion of penumbra observed by HINODE/SOT/SP. In such a case the ambiguity resolution is immediate, which enables the derivation of the horizontal gradient. Their inversion code was SIR, which provides height variations and then the vertical gradient. They plotted the histogram of the measured $\operatorname{div} \vec{B}$ with the result that the histogram is clearly not centered on 0 , but on $0.2 \mathrm{G} / \mathrm{km}$ instead. As this 0.2 value corresponds to the shift of the histogram but not to its width, Puschmann et al. (2010) pointed out that the 0.2 does not result from noise.

Faced with this observational result, we proposed a model based on plasma properties that is able to explain a departure from zero of $\operatorname{div} B$. This model is described in the second part of Bommier (2013) and Bommier (2014), and is recalled in Sect. 2.3 of the present paper. It is based on Debye shielding, but made anisotropic by the strong atmospheric stratification due to gravity. Our intuition was that the difference between vertical and horizontal gradients is related to the fact that horizontal and vertical characteristic lengths are different due to the stratification, itself due to gravity, but how does one relate the magnetic field to gravity? We found that Debye shielding is the intermediary. The present paper is devoted to a discussion of the question of departure from zero of $\operatorname{div} B$ according to different often raised questions: can the lack of spatial resolution be responsible for the observed phenomenon (Sect. 2.1)? It is known that in a medium with polarizability the magnetic field, often denoted as $\vec{H}$, may differ from the magnetic induction, denoted as $\vec{B}$, which has the property of $\operatorname{div} \vec{B}=0$. Thus, what are we measuring: $\vec{H}$ or $\vec{B}$ (Sect. 2.2)? In Sect. 2.4, after having recalled the stratification characteristics of the photosphere, we discuss the possible sources of non-zero div $\vec{B}$. Sect. 2.5 is devoted to present an experiment, where we had the impression during a presentation, that $\operatorname{div} \vec{B}$ would also be non-zero, also due to anisotropic Debye shielding. 


\section{Discussion}

\subsection{The non-zero div $\vec{B}$ cannot be ascribed to the lack of spatial resolution}

The departure from zero of the observed $\operatorname{div} \vec{B}$ value is often a priori ascribed to the lack of spatial resolution. In fact, it can be shown that this cannot be the case. The lack of resolution acts as filtering, and it can be shown that the divergence applied to the filtered quantity (the observed divergence) is equal to the filter applied to the local divergence. Consequently, if the local divergence is zero, the observed divergence would also be zero. Equivalently, if the observed divergence is non-zero, the local divergence is non-zero. The demonstration in terms of convolution products can be found in Bommier (2013) and in Bommier (2014), but the demonstration is yet easier in the spatial Fourier space because in Fourier transform, convolution products are transformed into ordinary products. Besides, in Fourier transform, the spatial derivative is transformed into a product by the spatial wave vector. The two products (resulting from the convolution and from the spatial derivative) are commutative, which leads to the result quoted above about the commutation between filtering and divergence. This demonstration is quoted in Bommier (2014).

In Bommier (2014), we report also the result of several tests, which were aimed to demonstrate that filtering may distort the divergence evaluation. Very sophisticated filtering schemes were considered, probably even more complicated than in reality. The filtering schemes were applied to a dipolar magnetic field of zero local $\operatorname{div} \vec{B}$. The filtered divergence never departed from zero, when the local divergence was zero.

It may be remarked that the measured quantities are however the Stokes parameters, and not directly the magnetic field. But the magnetic field is derived from the Stokes parameters by the inversion, which now involves all the parameters at the same time, and does not seaparate linear and circular polarization. These are known to behave quadratically or linearly with respect to the field, respectively, but in weak fields, and in sunspots the field is not weak. In the UNNOFIT inversion evaluation (Bommier et al. 2007 ), it was shown that what is determined is the local average magnetic field $\alpha B$, which is the product of the magnetic field strength $B$ by the magnetic filling factor $\alpha$. As the result of the measurement is the field averaged on the surface element, the measurement can be considered as spatially linear in magnetic field, so that the result above stated about the fact that spatial derivative and filtering may commute applies to the magnetic field measurement.

\subsection{What is measured: $\vec{H}$ or $\vec{B}$ ?}

It is well known that, in material media, one has to distinguish between the magnetic induction, denoted as $\vec{B}$, and the magnetic field, denoted as $\vec{H}$. This is the induction that obeys the Maxwell equation $\operatorname{div} \vec{B}=0$, but the divergence of $\vec{H}$ may eventually depart from 0 . The question then arises to determine what is really measured by the Zeeman effect: $\vec{B}$ or $\vec{H}$ ? In anisotropic media, $\vec{H}$ and $\vec{B}$ are related by $\vec{B}=\mu_{0}(\vec{H}+\vec{M})=\mu \vec{H}$, where $\vec{M}$ is the magnetization, and represents in some way the magnetic field generated by the electric charges turning about the field but remaining bound, so that $\vec{H}$ would be an external or large-scale field, whereas $\vec{B}$ would be the total, or local, or microscopic field (see Jackson 1975). The magnetization level is given by the magnetic susceptibility $\chi=\left(\mu-\mu_{0}\right) / \mu_{0}$. For bound charges contributing to the susceptibility, it was evaluated in Bommier (2013) and Bommier (2014) and found to be very small in the photosphere, on the order of $10^{-10}$. But there may be also a contribution of the free charges in the plasma, which was ignored in these two papers. The magnetic moment of a free charge 
of mass $m$ and velocity $\vec{v}$ is given by

$$
\vec{M}=-\frac{m v_{\perp}^{2}}{2 B} \vec{b},
$$

where $v_{\perp}$ is the velocity component perpendicular to the magnetic induction, and $\vec{b}$ is a unit vector parallel to the magnetic induction (Meyer-Vernet 2007, p. 63). The minus sign indicates the diamagnetic character of this magnetization due to the free charges. However, the numerical evaluation of this magnetization level in the solar photosphere leads to a susceptibility on the order of $10^{-5}$, also very small. Consequently, in the solar photosphere $\vec{H}$ and $\vec{B}$ are not different, within $10^{-5}$.

Indeed, it is well-known that the Lorentz force, which is at the origin of the Zeeman effect, is determined by the magnetic induction $\vec{B}$ and not the magnetic field $\vec{H}$ : for a charge $q$ of velocity $v$, the force is $\vec{F}=q \vec{v} \times \vec{B}$ (and not $q \vec{v} \times \vec{H}$ ). Eventually, in an heterogeneous medium with currents going through, a contribution would have to be added to this force, corresponding to a magnetic pressure gradient force due to a gradient of the permeability $\mu$. This contribution is locally

$$
-\frac{H^{2}}{2} \vec{\nabla} \mu
$$

(Bruhat 1967, p. 462). But, in the photosphere and on the atomic size, this contribution is totally negligible, being $10^{-33}$ times weaker than the magnetic (Lorentz force) contribution to the atomic hamiltonian, from which the Zeeman effect is studied.

Thus, one is faced with the difficult problem that what is measured with the Zeeman effect is really $\vec{B}$, the one that in principle obeys the Maxwell equation $\operatorname{div} \vec{B}=0$. It is generally assessed that only magnetic monopoles would be cause of $\operatorname{div} \vec{B} \neq 0$. The magnetic monopoles are a conjecture by Dirac (1931), but all the authors write as Jackson (1975) that "there is no experimental evidence for the existence of magnetic charges or monopoles". If a monopole had been found, one would have observed $\operatorname{div} \vec{B} \neq 0$. Because, on the contrary, one has always observed that $\operatorname{div} \vec{B}=0$, one concludes that monopoles are not observed. In other words, $\operatorname{div} \vec{B}=0$ is a result of observations, but limited only to prior observations, and the 3D magnetic mapping of the surface of the Sun is only a very recent field of investigation.

\subsection{Our proposal: the anisotropic Debye shielding}

In a medium, the magnetic field may be due to an external source, or to the electric currents made of charges moving in the medium. In the second situation, the magnetic field is expressed as a function of the electric currents, by the Biot \& Savart integral. As on the one hand, the solar observations conclude to a non-zero div $\vec{B}$ in sunpots, and as on the other hand $\operatorname{div} \vec{B}=0$ is and immediate and mathematical consequence of the Biot \& Savart integral, we were led to look for a phenomenon able to mask the field or make it disappear. This is the way by which we proposed a plasma effect, the anisotropic Debye shielding, as a possibility for explaining the solar observations. Our proposal is described in details in Bommier (2013) and Bommier (2014), and we will only summarize it here. The physical mechanism at the basis of the Debye shielding is as follows: given an electric charge in the plasma, the free charges of opposite sign come about it forming like a cloud of opposite charge, so that at a certain distance of the charge of interest, it is electrically masked by the cloud and there is no more electric effect due to the charge of interest. This is well-known for the electric field created by the charge of interest, but was not so clear for the magnetic field. In Bommier (2013) and Bommier (2014), we show that if the fluid velocity is constant over the cloud size (which, in the photosphere, is on 
the order of $2 \times 10^{-6} \mathrm{~m}$ ), the cloud acts also as a mask for the magnetic potential due to the charge of interest submitted to the fluid velocity. Thus, the magnetic field due to the electric currents in the medium is also submitted to the Debye shielding. The modeling of the Debye shielding is generally achieved in a rough manner, by considering that the electromagnetic range of the charge of interest is limited to the "Debye length". The Biot \& Savart integral has then to be limited to the Debye radius.

But we show in Bommier (2013) and Bommier (2014) that the Debye shielding in itself is not sufficient to explain a non-zero $\operatorname{div} \vec{B}$. If the shielding is isotropic, i.e. if the Debye volume is a sphere, when limiting the Biot \& Savart integral to the Debye length (identical in the three dimensions), div $\vec{B}$ remains 0 . This is only when one would have different Debye lengths in different directions, that one could have $\operatorname{div} \vec{B} \neq 0$. In a recent paper, Meyer-Vernet (1993) shows that the Debye length, generally expressed as a function of the gas temperature, is in fact determined by the particle velocities, thus extending the Debye length evaluation to non-LTE media. Remarking that in a surface layer as the photosphere, the particle velocity is highly anisotropic due to the stratification, we deduced that the Debye length is accordingly highly anisotropic also, leading to the possibility of non-zero $\operatorname{div} \vec{B}$.

Limiting the Biot \& Savart integral following the anisotropic Debye length, leads to a remaining possibly non-zero term in the $\operatorname{div} \vec{B}$ evaluation. But what about the electric currents, given by curl $\vec{B}$ ? We obtain that when the Biot \& Savart integral is limited to the Debye sphere, and when it is a sphere, $\operatorname{div} \vec{B}=0$ but curl $\vec{B}=\frac{2}{3} \mu_{0} \vec{J}$, and, on the contrary, when the Debye shielding is anisotropic, div $\vec{B}$ may be non-zero, whereas curl $\vec{B} \rightarrow \mu_{0} \vec{J}$ when the anisotropy increases.

The anisotropic Debye shielding effect is the way by which gravity, which is at the origin of the surface strong stratification, modifies the magnetic field.

Indeed, the velocity of a charged plasma particle is made of three components, namely: the thermal velocity, the fluid velocity, and an electromagnetic velocity resulting from the action of an eventual electric field on the charged particle. These components have to be added to form the particle velocity and all the effects studied in this paper (shielding, divergence and curl) behave linearly. It was already pointed out in Bommier (2013) and Bommier (2014) that the thermal velocity component does not contribute to any magnetic field vector or density current, because its average vanishes. But the eventual electromagnetic component was ignored in Bommier (2013) and Bommier (2014). This electromagnetic component is characterized by the fact that the electron and positive ion velocities are different. As a consequence, in the Debye shielding the velocity of the cloud is different from the one of the particle under interest. Thus an unshielded magnetic field remains due to the velocity difference. But, because it is not shielded, the divergence of this specific field is zero, as a result of the Biot \& Savart integral. Finally, the field resulting from the electromagnetic velocity component of the charged particle does not contribute to any non-zero $\operatorname{div} B$, whatever this electromagnetic velocity component be.

\subsection{The photosphere: a strongly stratified viscosity affected medium}

The stratified flows have been studied by Brethouwer et al. (2007). We evaluated the different numbers introduced by these authors, in the solar photosphere. The results are given in Tables $1 \& 2$ of Bommier (2013), where it can be seen that the horizontal Froude number is found $F_{h} \approx 0.02 \ll 1$, in the photosphere. As a consequence, the photosphere is a strongly stratified medium (this is not the case of the Solar Corona where the Froude number is found to be larger than 3). The Reynolds number is found to be $R_{e} \approx 400 \gg 1$, but the buoyancy Reynolds number is $R_{b}=R_{e} F_{h}^{2} \approx 0.1 \ll 1$, which implies that the 
photosphere is in the viscosity affected flow regime described by Brethouwer et al. (2007), which means in particular that no intertial cascade can develop (see their discussion of this regime). In this case, the aspect ratio between the horizontal and vertical typical lengths is given by $\sqrt{R_{e}} \approx 20$. The horizontal and vertical velocities are also different, so that the respective Debye lengths are different, leading to the anisotropy responsible for the observed non-zero $\operatorname{div} \vec{B}$.

But, as visible in Figure 3 of Bommier (2013) where a map of $\operatorname{div} \vec{B}$ in a $\delta$-spot was plotted rendering the spot shape instead of pure noise, which would have corresponded to a zero $\operatorname{div} \vec{B}$, the map of the $\operatorname{div} B$ scaled following the aspect ratio (i.e. by multiplying the horizontal derivatives by the aspect ratio) corresponds to a noise map (the $\delta$-spot shape is no more visible), which means that the $\operatorname{div} \vec{B}$ scaled by the aspect ratio becomes zero again.

The Debye shielding is an alternative to the magnetic monopole for magnetic flux modification. If there is a monopole, consequently $\operatorname{div} \vec{B} \neq 0$, but the inverse is not true. This is an implication, but not an equivalence. In other words, a monopole is a sufficient condition for $\operatorname{div} \vec{B} \neq 0$, but not a necessary condition. Existence of a monopole is not the only possibility for making $\operatorname{div} \vec{B} \neq 0$. A monopole would be responsible for magnetic flux creation. In the present paper, we worked on the opposite possibility, the magnetic flux disappearance by shielding. The symmetry is not total, because the shielding in itself is not sufficient to obtain $\operatorname{div} \vec{B} \neq 0$. The condition responsible for $\operatorname{div} \vec{B} \neq 0$ in the solar photosphere is the surface anisotropy resulting from gravity.

\subsection{An experimental evidence?}

In a seminar presented by Walter Gekelman on 2013 January 11th at Meudon Observatory, we heard something which could be some experimental evidence for non-zero $\operatorname{div} \vec{B}$ due to an anisotropy. This was about the Large Plasma Device (LAPD) at the Basic Plasma Science Facility (BAPSF) at UCLA. This experiment is made of a highly anisotropic plasma of length $L=18 \mathrm{~m}$ and thickness $l=0.6 \mathrm{~m}$. The characteristics of the plasma are electron density $n_{e}=10^{12} \mathrm{~cm}^{-3}$, electron temperature $T_{e}=6 \mathrm{eV}$ $=7 \times 10^{4} \mathrm{~K}$, axial magnetic field $B=200 \mathrm{G}$, but the three coordinates of the magnetic field were separately measured in the plasma. With these characteristics, we obtain as Debye length $L_{D} \approx 2 \times 10^{-5} \mathrm{~m}$, which is very small with respect to the plasma sizes, so that the Debye volume is entirely contained in it. We heard that it was necessary to correct the measured magnetic field coordinates by a factor up to 6 to recover $\operatorname{div} \vec{B}=0$. The necessity of the correction was ascribed by the presenter to misalignement of the three magnetic field coordinate detectors, but we think that the factor up to 6 is too large for this. We guess that in such an elongated plasma, the Debye sphere is also elongated, then anisotropic, so that in resemblance with the solar photosphere, here also one has $\operatorname{div} \vec{B} \neq 0$. The anisotropy is not completely similar to the one of the photosphere: in the photosphere, the Debye sphere is flattened as a disk. Here, the Debye sphere would be elongated as a cigar.

The description of the experiment is taken in Gekelman et al. (1991), and the physical conditions of the experiment are taken from Van Compernolle et al. (2014).

\section{Conclusion}

We have shown that magnetic monopoles are not the only possible cause for a non-zero $\operatorname{div} \vec{B}$, as it was observed in the solar photosphere. We have proposed the anisotropic Debye shielding as the possible cause of this observation, the cause itself being the anisotropy. The last cause of the phenomenon is the gravity, because the anisotropy 
is the one present at the surface of any star. The anisotropic Debye shielding is the way by which gravity, which is at the origin of the surface strong stratification, modifies the magnetic field.

We show in addition that when the components entering $\operatorname{div} \vec{B}$ are scaled following the stratified atmosphere aspect ratio, $\operatorname{div} \vec{B}$ becomes zero again, which would lead to a renewed MHD strategy for the solar photosphere, which was presented in conclusion of Bommier (2013) and Bommier (2014):

- $\operatorname{div} \vec{B}$ has to be computed with the coordinates scaled following the aspect ratio. For the rescaled $\operatorname{div} \vec{B}$, one has $\operatorname{div} \vec{B}=0$.

- curl $\vec{B}$ has to be computed without any modification. We have shown in Sect. 2.3 that when the anisotropy is sufficiently large (as it is in the solar photosphere), curl $\vec{B} \rightarrow \mu_{0} \vec{J}$, which is the usual Maxwell equation, in the photosphere where $\mu=\mu_{0}$ within $10^{-5}$.

For the divergence, this is true provided that the contribution to the magnetic field due to the electromagnetic component of the charged particle velocity submitted to an eventual electric field, leading to different velocities for electrons and positive ions, remains small with respect to the contribution of the fluid velocity. In other words, this is true provided that the velocity difference between electrons and positive ions remains small. Meyer-Vernet (2007, p. 86) states that in plasmas this difference is slight. For the curl, this is true in any case, provided that the stratification is strong, as in the solar photosphere.

\section{Acknowledgements}

I am very grateful to Bruce Lites, who was the referee of this paper, for kind and relevant improvements made to my manuscript.

\section{References}

Bommier, V. 2013, Physics Research International vol. 2013, Article ID 195403, 16 pages, doi:10.1155/2013/195403, http://www.hindawi.com/journals/physri/2013/195403/

Bommier, V. 2014, Comptes Rendus Physique 15, 430

Bommier, V., Landi Degl'Innocenti, E., Landolfi, M., \& Molodij, G. 2007, A\&GA 464, 323

Brethouwer, G., Billant, P., Lindborg, E., \& Chomaz, J.-M. 2007, Journal of Fluid Mechanics 585,343

Bruhat, G. 1967 Cours de Physique Générale: Électricité, Masson

Dirac, P. A. M. 1931, Royal Society of London Proceedings Series A 133, 60

Faurobert, M., Aime, C., Périni, C., et al. 2009, A\&A 507, L29

Gekelman, W., Pfister, H., Lucky, Z., et al. 1991, Review of Scientific Instruments 62, 2875

Grec, C., Uitenbroek, H., Faurobert, M., \& Aime, C. 2010, A\&SA 514, A91

Jackson, J. D. 1975, Classical Electrodynamics, 2nd Edition, Wiley \& Sons

Khomenko, E., \& Collados, M. 2007, ApJ 659, 1726

Leka, K. D., Barnes, G., Crouch, A. D., et al. 2009, Solar Phys. 260, 83

Metcalf, T. R. 1994, Solar Phys. 155, 235

Meyer-Vernet, N. 1993, American Journal of Physics 61, 249

Meyer-Vernet, N. 2007, Basics of the Solar Wind, Cambridge University Press, Cambridge

Puschmann, K. G., Ruiz Cobo, B., \& Martínez Pillet, V. 2010, Ap. Lett. 721, L58

Van Compernolle, B., Bortnik, J., Pribyl, P., et al. 2014, Physical Review Letters 112, 145006 